DOI: $10.2478 / \mathrm{v} 10122-012-0007-\mathrm{Z}$

\title{
A COMMUNICATIVE COMMUNITY OF AGENTS
}

\author{
DOROTA LIPOWSKA
}

\begin{abstract}
Dorota Lipowska. A Communicative Community of Agents. Lingua Posnaniensis, vol. LIV (1)/2012. The Poznan Society for the Advancement of the Arts and Sciences. PL ISSN 0079-4740, ISBN 978-83-7654-103-7, pp. 77-87.

Computer modelling is becoming an increasingly important tool for researching the problem of origin and evolution of language. A fundamental technique is that of multi-agent modelling, which simulates a system of dynamically interacting individuals called agents, equipped with strictly defined properties and rules governing their behaviour or evolution. In such a population (a communicative community), as a result only of local interactions between agents, a process of self-organization occurs and some kind of global property emerges, such as linguistic coherence. Presented here are two models of the naming game type, in which agents exchange names of objects, gradually establishing a common vocabulary. In the evolutionary version there was observed a very strong link between biological and linguistic processes, being a clear manifestation of Baldwin's effect - genetic assimilation of the ability to learn (a language, for example). In the multi-object version the development of homonymy and synonymy was studied, as well as the effect of noise on a developing language.
\end{abstract}

Dorota Lipowska, Institute of Linguistics, Adam Mickiewicz University, al. Niepodległości 4, PL 61-874 Poznań, lipowska@amu.edu.pl

\section{INTRODUCTION}

The concept of communicative community as introduced by Ludwik Zabrocki (for a list of his works on this subject see BAŃCZEROWSKI 1980: 18) is an important category used in sociolinguistics and in research into language history and change (e.g. ZABROCKI 1963; BORAWSKI 2005). A communicative community refers to a group of people striving to exchange information, irrespective of the means used to achieve that purpose. Hence within a single community there need not even exist a method of communication common to all members - there may be multiple such methods, and in particular there may be multiple languages in use.

The present article, however, is not about either sociolinguistics or language history, but rather addresses the "prehistory" of language, and from a quite abstract viewpoint. Namely, the author is interested in the use of computer simulations to investigate the problem of the origin and evolution of language, and in particular the use of multi-agent simulations to implement various versions of language game models. In presenting the systems examined by the author, the term "communicative community" will be used in relation to a simulated set of agents, although it is understood that this usage is rather metaphorical. Before proceeding 
(in sections 2 and 3) to present the author's results, an attempt will first be made to explain why computer simulations are useful in this field.

In the last two decades there has been a huge growth in interest in the problem of the origin and development of language. A feature of this research is that, while many different theories are proposed, there is virtually no empirical base. The origin of language is an event so distant in time that it has left no direct traces. The phenomenon is not a repeating one, and so cannot be a subject of our observations. There remains evidence relating to processes such as language acquisition in children and second-language learning by adults, the formation of pidgins and creoles, and possibly animal communication, but these phenomena, although related, are of a different kind. Moreover not only is there an absence of empirical evidence enabling the verification of proposed hypotheses, but the hypotheses are also often formulated in such general terms that it is not possible in fact to specify any detailed and verifiable predictions based on them.

We currently have at our disposal a new method of formulating scientific theories: we can present them not only verbally or symbolically, but also in the form of computer programs, and computer simulations are an excellent method of testing research models. Programs are written based on hypotheses concerning the causes, mechanisms and processes of the simulated phenomena, which must therefore necessarily be formulated explicitly and in a manner that is sufficiently detailed, clear, precise and non-contradictory. As D.E. Knuth put it: "Science is what we understand well enough to explain to a computer." These hypotheses can then be tested comprehensively under the conditions of virtual experiments. In a situation where there is no direct empirical evidence or observation, as is the case when studying the problem of language origin and development, a tool such as computer simulation would appear to be especially valuable.

Language can be described at an individual or population level, and in studying its origins we cannot overlook the question of the evolution of its users. The processes acting at these different levels operate on different time scales (ontogenetic, glossogenetic, phylogenetic); however, they are not independent, but have a mutual influence on one another. Therefore for an adequate description of language origin and evolution there is a need for very complex models, and the task of presenting these in an analytical manner would seem to be formidable, if not impossible.

The origin of complex systems is often successfully explained as the result of processes of adaptation and self-organization. A group of people striving to communicate with each other (a communicative community) can also be treated as a complex adaptive system, namely a population of separate individuals in which, without any global control and purely as a result of their local interactions, the system self-organizes and some global property emerges in it (linguistic coherence in this case). Hence it is an assumption of this approach that language is passed on not (only?) genetically, but culturally, through learning from other individuals. It can be expected that positive feedback - a characteristic feature of adaptive systems - will occur here (for example, between mastery of a certain linguistic convention and the frequency of its use). Hence in this viewpoint the development and growth of complexity of language is regarded as an effect of its cultural transmission and adaptation ("glossogenetic evolution").

An excellent technique for simulating adaptive systems is that of multi-agent modelling, which makes it possible to investigate a problem in a synthetical manner (bottom- 
up method). A system of dynamically interacting individuals called agents is simulated. Agents are equipped with strictly defined properties and rules governing their behaviour or evolution. As a result of many local interactions between agents or between agents and the environment, global properties of the population emerge. This technique can be used successfully for studying dynamic complex systems (and language is undoubtedly such a system), for which it is difficult to apply a classical analytical (top-down) research approach.

In studies of the evolution of language, two main types of such models can be distinguished. The first of these is the iterated learning model. Here language is passed on between successive generations: agents of the younger generation learn the language on the basis of utterances presented to them by agents of the older generation, after which a generational replacement takes place - the older generation is replaced by younger agents, and these in turn are replaced by a new generation, this process being repeated many times (KIRBY 2002). Simulations of this type have been used mainly to study the evolution of holistic language into compositional language. However, to investigate issues of the emergence of various aspects of linguistic coherence within a population (and hence the formation of a communicative or language community) a second type of model is generally used - the language game model. Here there is no division into older and younger agents nor generational replacement, but agents engage in interactions known as language games (STEELS 1995, 1997). An example of such games is the naming game, in which agents exchange various names of objects, gradually establishing a common vocabulary. Two models of this type will be described below.

\section{THE EVOLUTIONARY NAMING GAME MODEL}

The first of the models is an evolutionary version of the naming game (LIPOwSKI \& LIPOWSKA 2008; LIPOWSKA 2011). In this model we consider a set of agents situated on the vertices of a square lattice. The agents try to establish a common word for one object (the assumption that their communication concerns a single object appears not to limit the generality of the considerations, and is often used in models of this type). They achieve this by playing, in pairs, successive instances of the naming game. A randomly selected agent becomes the speaker, and communicates one word from its lexicon (inventory of words) to a listener, which is selected at random from the speaker's nearest neighbours. The game ends in success if the listener knows the word (has it in its lexicon), and in failure otherwise. According to the result, the listener and speaker modify their lexicons, namely lists of words to which weights (positive numbers) are assigned. The speaker selects words for communication with a probability proportional to their weights. Moreover every agent has an also numerically defined language learning ability, which is used in the modification of its lexicon: in the success case both speaker and listener increase the weights of the communicated word by the value of their respective learning abilities, whereas in the failure case the speaker reduces the weight of this word in the same manner (and if the weight becomes negative, removes the word from the lexicon), while the listener adds the word to its lexicon with unit weight.

Agents not only communicate with each other, but also evolve. At every step of the simulation, with a set probability of communication, the selected agent either becomes the speaker or undergoes certain life processes: it dies or reproduces, possibly with mutation. 
The probability of the agent's survival (the measure of its fitness) is defined by a formula that takes account of both its age and its linguistic performance (measured by the sum of weights in the lexicon), i.e., fitness decreases with age, but increases with performance. An agent that survives will breed providing there is a free neighbouring vertex. The offspring generally inherits the parent's learning ability and its language (the word with the highest weight). With a certain small probability mutations occur and the offspring takes on a new (randomly selected) value for learning ability or a new word.

Simulations of this model begin from a configuration where all sites of a lattice are occupied by agents with randomly determined learning abilities and single words. The parameter of greatest importance for the behaviour of the model is the probability of communication, which determines the frequency of acts of communication relative to population changes. If this value is small, the model remains in the phase of "linguistic disorder" with only small clusters of agents using the same language (where an agent's language is defined here as its word of the highest weight; this means that agents using the same language usually - though not necessarily always - communicate with each other using a mutually recognizable word, which assures them of a relatively large number of successes). As the probability of communication increases, the clusters slightly grow (this process is called coarsening); but only when a certain threshold value is exceeded, is there a rapid qualitative change and the model suddenly enters the phase of "linguistic coherence", in which almost all agents speak the same language. Interestingly, the dependence of the agents' lifetime on their language learning ability is also different in these two phases: only in the singlelanguage phase are these values markedly correlated (the greater the ability, the longer the lifetime), while in the multi-language phase they remain virtually independent. This can be explained by noting that in a linguistically stable environment faster learning enables faster adaptation, and hence greater fitness, while in a chaotic environment it does not provide any real benefits, since it will not in any case significantly increase the number of successes.

The occurrence of a significant change in the system when the probability of communication exceeds a certain threshold value was confirmed by measurement of average values of the number of agents' successes and their learning abilities: both parameters increased rapidly at the same critical point. This co-occurrence means that in this model the linguistic factors (communication success) and biological factors (innate abilities) are very strongly linked; for this reason we refer here to a biolinguistic transition.

The nature of this process changes somewhat when it is assumed that the probability of communication in the model is subject to continuous growth (justified in that, as the human species developed, verbal communication undoubtedly became increasingly important and communicative acts gradually became more frequent). Simulations of the model with the frequency of communication increasing over time showed that in this situation, instead of one sudden transition, there occurs a series of changes, also quite rapid, but involving smaller jumps. For example, if the system starts with minimum learning ability values selected randomly for agents (which seems to be a reasonable assumption for the initial state, where there was still no language), it fairly rapidly reaches a state which is homogeneous in this respect - where almost all agents have similar (though generally quite small) abilities. With time and with the gradual increase in the probability of communication these small abilities become clearly inadequate, and suddenly rise quite quickly, after which, after transitional 
disturbances, the system again becomes homogeneous in this respect. However, it still lacks linguistic coherence - there are very many different languages used, each by only a small number of agents. After a certain amount of time a further transition takes place and the system enters a phase in which maximum learning abilities dominate. At the same time the level of average communication success also rises rapidly, and the number of languages used decreases, meaning that many of them die out while others become more and more widely used. Finally the system reaches a linguistically homogeneous (mono-language) state, with an almost $100 \%$ level of communication success, although this happens only after a very long time. We should emphasise that this change in the character of the model's evolution (a series of transitions instead of a single large one, i.e. multi-step rather than single-step dynamics) is a consequence of continuous change in the probability of communication. In turn the rate and character (continuous or discrete) of change in communication frequency can be interpreted as the rate and manner of occurrence of cultural changes. As can be seen, our model suggests that cultural factors also have a huge influence on the course of language evolution. In summary, it can be concluded that the proposed version of the evolutionary naming game incorporates the three principal factors affecting language development: learning, culture and evolution. It is possible, even, that it was this combination of three factors and their interaction and feedback relations that triggered the rapid development of the human species.

Let us look at the issue of language learning somewhat more closely. An agent increases its number of successes by learning the languages of its neighbours. Greater communication success leads in turn to greater fitness, namely higher chances of survival. It is therefore beneficial to possess large innate learning abilities - and indeed we can observe a gradual growth in the level of these abilities in the population. How, though, can this influence of individual learning on the genetic adaptation of a whole population be explained within the framework of Darwinian evolution, considering that offspring do not inherit the skills acquired by their parents? Apparently in our model, the Baldwin effect manifests itself(WEBER \& DEPEW 2003). At a sufficiently high probability of communication, interactions take place sufficiently often to produce clusters of agents speaking the same language, which gives them a large number of successes. Such clusters of agents with high linguistic attainments (these being the result of learnt behaviour) may be regarded as ecological niches which steer the evolution by favouring individuals with high learning ability. Therefore it is not the learnt behaviour itself that is inherited, but the ability to acquire it; and thus a population gradually emerges in which the beneficial abilities that were initially only acquired become, over time, innate and instinctive. This is the mechanism of the Baldwin effect, i.e. of genetic assimilation.

The positive feedback present in the model (given a sufficiently large probability of communication, high language learning abilities facilitate communication to such an extent as to produce sufficiently large and stable linguistically coherent clusters to favour the further development of language abilities) leads in the end to the aforementioned biolinguistic transition: the population experiences an abrupt rise in the average values both for communication success and for learning ability. It can be asked, of course, how far such behaviour of the model reflects the features of natural language evolution. The development of language, which probably began approximately 100,000 years ago, went hand in hand with 
significant anatomical and genetic changes (such as lowering of the larynx, enlargement of the brain and fixation of the FOXP2 gene), which undoubtedly favoured that development. But what initiated this process? Presumably the emerging lifestyle produced a greater and greater need to exchange information, and this stimulated the gradual formation of a common repertoire of means of communication. No doubt even in these archaic populations there occurred certain linguistic and cultural interactions that influenced our ancestors' evolution, inter alia, via the aforementioned Baldwin effect.

To return to our model, might it thus be within the proper bounds of terminology to call its population of agents a communicative community? After all, the probability of communication is a measure of the need for mutual understanding; this need, according to L. ZABROCKI (1963: 12), determines the basis and essence of the communicative community, whose existence begins when that need is concretely realized. Further: "The production of a common means of communication within a given communicative community is part of the essence of the communicative community. Every communicative community strives to produce a common means of communicating. Every communicative community must strive to eliminate differences relating to means of communicating." It is this type of situation that arises in our model. We are disregarding, of course, the nature of the bonds which might stimulate such a need for communication (whether they are social, economic, cultural, ideological, religious, emotional, etc.); we note that the corresponding parameter, the probability of communication, can be equally well interpreted as the strength of those bonds. In any case, provided it is sufficiently large, there begins to emerge within that community a common repertoire of linguistic means. When the probability of communication is small, we still have a loose community, which does not have such an integrating effect on the means of communication as does a tight community, which in turn emerges following an increase in the probability of communication. The greater this probability, the tighter the communicative community, although there are still many languages present within it. Finally, after a critical point is passed, the communicative community changes into a language community, in which almost all agents use the same language. Continuing to make the distinctions introduced by L. Zabrocki (ibidem: 14-15), we can regard the entire population of agents as a higher-level community, and the monolingual clusters (language communities) appearing within it as lower-level communities (being same-level communities towards each other). Moreover, if apart from an agent's "main" language (the word with the highest weight) we also take into account the other languages (words) known to it, it will turn out that an agent may belong simultaneously to different communicative communities (i.e., to the main community as well as to subsidiary communities). Some communities develop, while others disappear - and thus live communities may in time become extinct (even though they previously produced a common means of communication, after a certain amount of time they fail to demonstrate its circulation within the community as a whole). L. ZABROCKI (ibidem: 16-17) refers also to integrating and disintegrating processes in societies and in relation to languages. All integration in communicative communities causes the vanishing of dialects or languages; over time, linguistic integration results. In particular a higher-level community gradually eliminates lower-level communicative communities (it eliminates their languages). Integration of this type is also found in the model described here (as a coarsening process). 
It might therefore be concluded that the terminology introduced by L. Zabrocki to describe human social groups is ideally suited to the computer-simulated model of language origin and evolution as presented here, based on a set of inanimate agents. Is the model thus properly constructed, considering all described conceptual categories fit it and the corresponding linguistic phenomena occur within it? This would be a powerful argument in favour of its correctness and adequacy. Unfortunately, it is not hard to see that this fit is rather superficial. According to L. Zabrocki (ibidem: 9): "The reasons for the origin and vanishing of languages $[\ldots]$ are a result of the birth, life and disappearance of corresponding communicative communities. There are no immanent-linguistic laws here." Hence a primary role is played by social groups and their transformations (induced by economic, social, cultural and other factors), which trigger language changes. However, in the model no account is taken of the history and development of communities; the essence of the system is just the process of transmission of language between agents attempting to communicate. The dynamics of the model are based exclusively on the elementary act of communication, which turns out to be sufficient for language communities to form and vanish within the system. The agents are not equipped here with beliefs, goals or abilities from which their actions would result they are not intentional, active individuals, but rather passive carriers or relays of language, or vehicles as they are called by N. RiTT $(2004,2010)$. In spite of this, as a result only of multiply repeated attempts at communication, languages emerge, and their further development is a result solely of that interpersonal linguistic transfer (i.e. cultural transmission).

\section{THE MULTI-OBJECT NAMING GAME MODEL}

The emergence of language initiated cultural development of a cumulative nature, thanks to the inheritance of the achievements of previous generations by successive ones. It became possible for large groups - and not only family groups - to cooperate, which led to the formation of a very complex society. In the framework of Darwinian evolution, our willingness to share knowledge with relatives can be explained by kin selection, but it is not easy to find arguments to explain cooperation with unrelated individuals. Since speaking is a more costly process than listening (it takes time and energy, and in the past might have exposed the speaker to the risk of attack by a predator), selection ought to favour egoistic individuals oriented only towards receiving information and not transmitting it - but in that case language would not have developed at all. Hence the honest transmission of unfalsified information must nonetheless pay off in some way (for example by raising status within the group, increasing sexual attraction, enabling the manipulation of other individuals, etc.).

Computer simulations which are of interest in this context were carried out by J.R. HuRFORD (2003). He proposed an evolutionary version of an iterated learning model, similar in character to genetic algorithms. In this model the agents had innate predispositions to avoid (or not) synonyms or homonyms (i.e. an agent's genotype determined the corresponding manner in which that agent learnt the language of the previous generation of agents). The system evolved until one of the genotypes completely eliminated the others. Here the fitness of agents was determined based on their either communicative or interpretative success, defined respectively as the average probabilities that a given agent (as speaker) would be correctly understood by others or that it (as listener) would correctly understand other 
agents. The simulations showed that when selection was based on communicative success, the winning genotype was one that rejected synonyms but tolerated homonyms, but when evolution was controlled by success at interpretation, it was the opposite genotype, rejecting homonyms but tolerating synonyms, that emerged victorious. Naturally the languages that emerged corresponded to the dominant genotype, and thus only in the first case did there emerge languages similar to natural ones, in which complete synonymy is rare, but homonymy very common. As J.R. Hurford (2003: 450) concludes: "Humans evolved to be well adapted as senders of messages; accurate reception of messages was less important in our prehistory. We may be primarily speakers, and secondarily listeners."

Clearly, then, people are good senders of messages. However, the fact that synonyms are not numerous in natural languages, while homonyms occur quite frequently, is surprising in that synonymy ought not to affect the effectiveness of communication, while homonymy must in principle impair it. We may also note that in computer languages homonyms do not occur at all, although synonyms are permissible (and computers are ideal receivers of messages). It therefore seems possible to regard this clear asymmetry between rare synonymy and frequent homonymy in natural languages as a characteristic (generic) feature of those languages, and to use it as a test for computer models of language development.

This test seems to be passed successfully by the next model to be presented here (LIPOWSKI \& LIPOWSKA 2009). This is a multi-object version of the naming game. This time the model contains only two agents, alternately playing the roles of speaker and listener. We note, incidentally, that even such a minimal group can be called a communicative community, since to quote again L. Zabrocki (1963: 12), "a communicative community comes into being when the need for communication arises between at least two individuals" (and when a group consists of only two individuals, it is presumably more accurate to call it a community than a population). Indeed, as it turned out, by studying even such minimal communities one can obtain interesting results.

It is assumed that agents transmit to each other names of certain objects. For each of the objects, the agent has in its lexicon a list of words that refer to it, and to these words weights are assigned, which determine the course of stochastic processes both of selection of a communicated word by the speaker as well as of the determination of the meaning of that word by the listener. Initially, both agents have single random words on each list. The agents then play a series of instances of the naming game: the speaker selects an object at random, and then selects a word from the list for that object (according to the weights), which it communicates to the listener; the listener then attempts to guess the word's meaning. To do this, it first computes, for each list in its lexicon, a global measure of its similarity to the given word; each word on the list contributes to this measure according to how close it is to the given word and what its own weight is (an identical word increases the measure so much that it becomes practically certain that the list in question will be chosen). Next the listener (again in accordance with the computed measures) randomly selects a list, and thus also the object assigned to it. The game ends in success if by this means the listener succeeds in correctly establishing the meaning of the given word, or in failure otherwise. Both agents then make appropriate modifications to their lexicons. In the success case the weight of the word in question is increased (after the listener has added it to its appropriate list if it was not yet on that list but the list was still correctly chosen by the listener). In case of failure 
the speaker reduces the weight of the given word, while the listener accordingly adds it to the appropriate list or increases its weight if it is already there. This modification of weights corresponds to reinforcement learning.

The simulations showed that agents fairly rapidly correlate their lists in such a way as to enable them to communicate effectively (so as to achieve maximum communication success). A common language thus emerges, in which, as it turns out, the words with the greatest weights from each individual list ("highest ranked" words) are generally in use. As can be seen, in this model too, a communicative community transforms into a language community.

The agents "talk" about many objects, and hence the emerging language may contain homonyms and synonyms. Because the rules of the model contain numerous probabilistic elements, our definition of homonymy and synonymy should also take account of these. Hence a homonym is defined here as a word which may with relatively large probability refer to different objects, while synonyms are defined as words which may with relatively large probability refer to the same object. Since the agents generally use highest ranked words (very rarely those ranked second, and others almost never), this means that homonyms will be those words that occur on at least two of an agent's lists as highest ranked, while synonyms will be such pairs of words which both agents have on the same list (i.e., corresponding to the same object) with the highest and second highest weights.

This means that the number of different highest-ranked words is a measure of homonymy in the model: the greater the number, the fewer homonyms there are. The simulations showed that in the course of language evolution this number increases (similarly as the number of communication successes), approaching the maximum value, namely the number of objects in the system - although it never reaches that value, but stabilizes below it. This means that although in the course of language development a redistribution of words takes place which reduces homonymy, enabling more and more effective communication between the agents, nonetheless homonymy never disappears completely and seems to be a persistent feature of the language.

On the other hand, when the numbers of successes achieved using highest and second highest ranked words are analysed, it turns out that both increase over time (although the second increases more slowly). The use of second ranked words thus ensures a relatively large number of communication successes, which shows that indeed to certain objects there correspond (at least) two different words, which thus can be considered as synonyms. However, analysis of the frequency of use of second ranked words showed that this value decreases to zero over time (and all communication then takes place exclusively using highest ranked words), which means that in the course of the development of the language synonyms gradually go out of use, and after a sufficiently long time they disappear completely.

Both of these effects (rare but persisting homonymy on the one hand, and vanishing synonymy on the other) are in fundamental accordance with the situation observed in natural languages. As we have already mentioned, Hurford argued that this phenomenon is caused by the asymmetry between the evolutionary benefits to speaker and listener. In our model, however, the language develops only via cultural mechanisms, which involve exclusively the transfer of language between agents, and that within one generation only, without generational replacement. The results therefore show that certain elementary properties of 
homonymy and synonymy may be explained within a model which is much simpler than Hurford's evolutionary model and does not require any recourse at all to the process of natural selection.

In the same model, the effect of noise on language evolution was also studied. Sometimes, even when noise causes a word as received by the listener to differ slightly from that transmitted by the speaker, the listener is still able to interpret it correctly. This is possible if the amplitude of the noise is small (so the word is deformed only slightly), or if the highest ranked words on individual lists are sufficiently differentiated (so that the deformation of one of them will not cause it to overlap with another one). Hence the distribution of highest ranked words was also analysed. The simulations of a model without noise showed that in the course of language evolution there occurs a redistribution of those words in the available "verbal space" which reduces homonymy, and thus improves communication between agents. The noise introduced into the model has a significant effect on the further progress of that redistribution, leading to a more and more uniform distribution. Greater differentiation of words, however, means further reduction in homonymy. On the other hand, the noise also affected the distribution of second ranked words, in such a way that they became very close to the highest ranked words. It is hard to consider such close pairs to be typical synonyms; they correspond rather to something like morphological variants of the same word. The results therefore suggest that noise might also play an important role in the evolution of language, having a favourable effect on adequate separation of words and reducing homonymy and (possibly) synonymy.

Naturally both of the models described above provide only fragmentary suggestions as regards the problems of language evolution. There is undoubtedly a need for further research and for much more complex models to obtain a more complete understanding of the problem. The models described here disregard both the internal organization of the communicative communities and the relations between them, focusing exclusively on the processes of language learning and transmission. It may be suggested, therefore, that subsequent models should be built to take account of possible intra- and inter-community relationships.

\section{REFERENCES}

BANZHAF Wolfgang et al. (eds.). 2003. Advances in Artificial Life - Proceedings of the Seventh European Conference on Artificial Life (ECAL). Lecture Notes in Artificial Intelligence vol. 2801. Berlin: Springer-Verlag.

BAŃCZEROWSKI Jerzy. 1980. "Ludwik Zabrocki as a Theorist of Language.” In: ZABrocki 1980: 9-22.

BorawsKi Stanisław. 2005. "Podstawy idei poznawczej studiów nad dziejami używania języka polskiego. Esej o diachronii." In: BORAWSKI 2005: 13-61.

Borawski Stanisław (ed.). 2005. Rozprawy o historii języka polskiego. Zielona Góra: Oficyna Wydawnicza Uniwersytetu Zielonogórskiego.

Hurford James R. 2003. "Why Synonymy is Rare: Fitness is in the Speaker.” In: BANZHAF et al. 2003: 442-451.

KirBy Simon. 2002. "Natural Language from Artificial Life." Artificial Life 8(2), 185-215.

LiPOWSKA Dorota. 2011. "Naming Game and Computational Modelling of Language Evolution." Computational Methods in Science and Technology 17(1-2), 41-51.

Lipowski Adam, Lipowska Dorota. 2008. "Bio-linguistic Transition and Baldwin Effect in an Evolutionary Naming-game Model.” International Journal of Modern Physics C 19, 399-407.

Lipowski Adam, LipowsKa Dorota. 2009. "Language Structure in the n-object Naming Game." Physical Review E 80(5). 
Pinker Steven, Bloom Paul. 1990. "Natural Language and Natural Selection." Behavioral and Brain Sciences 13(4), 707-784.

RitT Nikolaus. 2004. Selfish Sounds and Linguistic Evolution: A Darwinian Approach to Language Change. Cambridge: Cambridge University Press.

RitT Nikolaus. 2010. "Agents or Vehicles? The Role of Speakers in Directing Linguistic Evolution.” Plenary talk delivered at 41st Poznań Linguistic Meeting PLM2010, 23-26 September 2010, Gniezno, http://ifa.amu.edu. pl/plm_old $/ 2010 /$.

SteEls Luc. 1995. "A Self-organizing Spatial Vocabulary.” Artificial Life 2(3), 319-332.

Steels Luc. 1997. "The Synthetic Modeling of Language Origins." Evolution of Communication 1(1), 1-34

Weber Bruce H., Depew David J. (eds.). 2003. Evolution and Learning - The Baldwin Effect Reconsidered. Cambridge: MIT Press.

ZABRoCKI Ludwik. 1963. Wspólnoty komunikatywne w genezie i rozwoju języka niemieckiego. Part I: Prehistoria języka niemieckiego. Wrocław-Warszawa-Kraków: Zakład Narodowy im. Ossolińskich.

ZABRocki Ludwik. 1980. U podstaw struktury i rozwoju jezzka. At the Foundation of Language Structure and Development. Warszawa-Poznań: PWN. 